\title{
Optical Properties of Te Thin Films Prepared by Thermal Evaporation Technique
}

\author{
Fatin G. Hachim \\ Department of Physics-College of Science- University of Thi -Qar.
}

\begin{abstract}
$\underline{\text { Abstract }}$
This work describes, Tellurium (Te) films were deposited on glass substrates at room temperature by the method of vacuum evaporation with thickness $(0.4 \mu \mathrm{m})$, with rate of deposition equal to $(5.5 \AA / \mathrm{sec})$, the samples are annealed in a vacuum for one hour at (373 and 433) K. The optical properties such as absorption coefficient $(\alpha)$ was determined using the absorbance and transmittance measurement from FTIR Shimadzu spectrophotometer, with range of wave length (2800-3900) $\mathrm{nm}$. And also calculated optical constants (refractive index (n), extinction coefficient (k)) for Tellurium films. The tests have been shown that the optical energy gap increases with increasing of annealing temperature for the samples.
\end{abstract}

Keywords: optical properties of Te films, effect of annealing on optical properties of Te films.

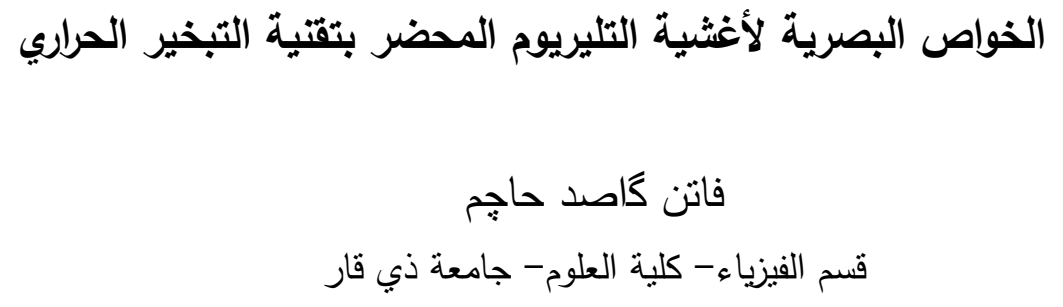

الخاخاصة

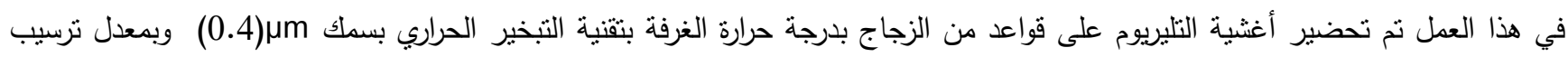

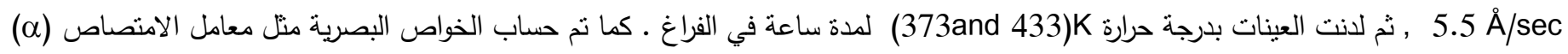
الذي حسب من خلال قياسات الامتصاصية والنفاية باستخدام جهاز

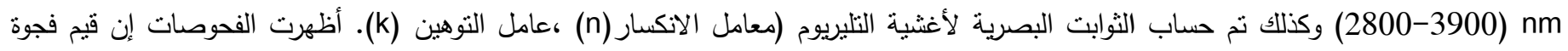
الطاقة للأغثية تزداد بزيادة حرارة التلدين.

\section{Introduction}

Tellurium belongs to the group of chalcogens, which also includes the elements oxygen, sulfur, selenium and polonium, It was first discovered by Mineralogist Franz-Joseph Muller Von Reichenstein in 1782[1]. Chalcogenide materials received much attention because of their important application in various solid state devices and in optical applications [2]. Te thin films have been extensively used in various technological areas, especially in microelectronic devices Such as gas sensor, optical information storage, 
All these applications are due to remarkable physical properties of Te such as low band-gap and transparency in the infrared region [3]. It has considered being an excellent candidate for use in future high-efficiency photoconductors, thermoelectric devices, $\mathrm{Te}$ have atomic number of 52 is one of the rarest elements in the earth crust. Tellurium is a narrow-band gap semiconductor with band gap energy of $0.35 \mathrm{eV}$ [4]. Commercial applications of Te include vulcanization of rubber, in alloys, use to color glass and ceramics [5]. The aim of the present work is to study some optical parameters like, transmission, absorption coefficient, energy gap, and optical constants. Also it have been studied the effect of the annealing on these parameters.

\section{Experimental}

Thermal evaporation method is used for obtaining thickness (0.4) $\mu \mathrm{m}$ of Tellurium thin films under vacuum pressure 10-5 mbar onto cleaned glass substrates. A molybdenum boat was used. The distance between the boat and the substrate is $15 \mathrm{~cm}$, Type Blazers Model [BL 510] was used to deposit Te thin films, an electric current was passed through the boat gradually to prevent breaking the boat, when the boat temperature reached the required temperature the deposition process starts. After these steps the current supply was switched off and the samples were left in the high vacuum, then the air was admitted to the chamber, and the films were taken out from the coating unit and kept in the vacuum desiccators until the measurements were made. The film thickness measured with an interference microscope. The parameters that control the nature of the film properties are thickness (0.4) $\mu \mathrm{m}$ and annealing temperature (373 and 433) K, The optical absorbance spectra of the Te films are measured using FTIR Shimadzu spectrophotometer model 8300, Japan, with range of wave length (2800-3900) $\mathrm{nm}$. The optical constants including, the refractive index, extinction coefficient were calculated from transmittance and absorptance spectrum.

\section{Results and Discussion}

The most absorption process involves the transition of electrons from the valence to conduction band, an electron absorbs a phonon (from the incident beam), and jumps from the valence band to conduction band. The photon energy must be equal to the energy gap or larger [6].

Relation the transmittance ( $\mathrm{T}$ ) with the absorbance (A) :

$\mathrm{A}=\log (1 / \mathrm{T})$

In the present study, Fig (1) show the variation the transmittance with different annealing temperature, the transmittance increases with increasing of annealing temperature for all samples. The absorbance decreases with increasing of annealing temperature, this behavior is opposite completely to that of the transmittance as shown in Fig.(2).

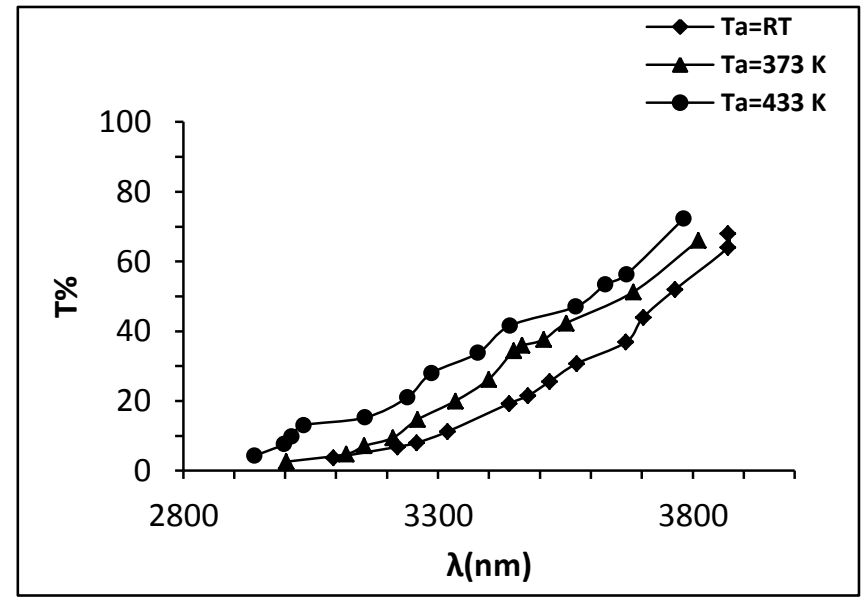

Figure (1): Transmittance spectrum as a function of wavelength for Te films at different annealing temperatures

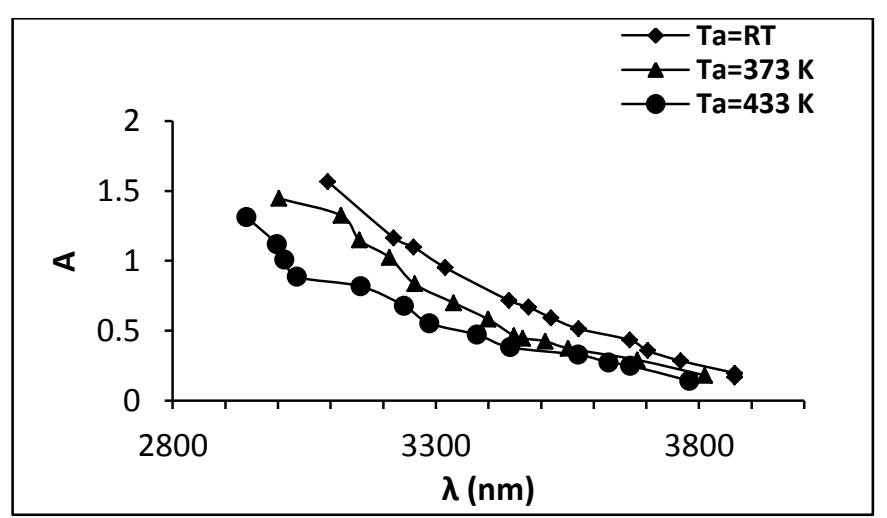


The relation used to given absorption coefficient $(\boldsymbol{\alpha})$ [6]:

$\alpha=2.303 \mathrm{~A} / \mathrm{t}$.

where $t$ is the sample thickness.

We can notice that $\alpha$ (absorption coefficient) decreases with increasing of $T_{a}$ for all films as shown in Fig. (3) because increasing energy gap, Also $\alpha$ has the same behavior of the absorbance.

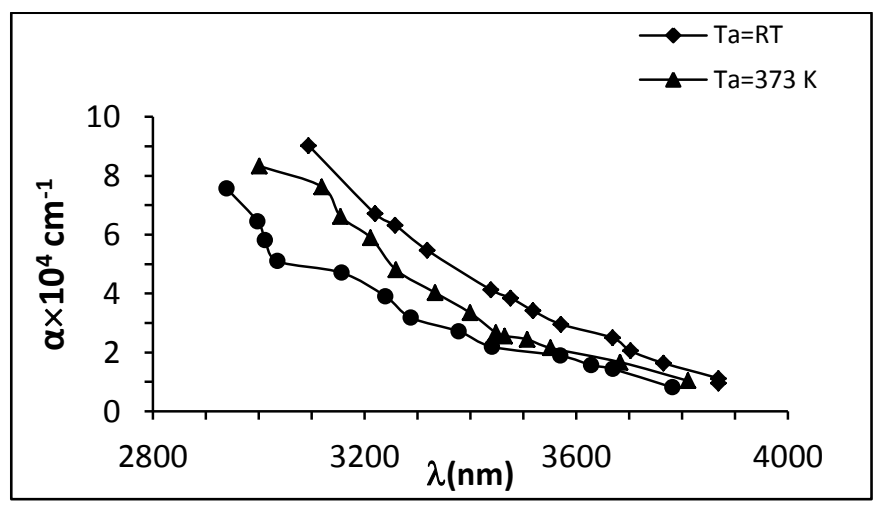

Figure (3): Absorption coefficient as a function of wavelength for Te films at different annealing temperatures

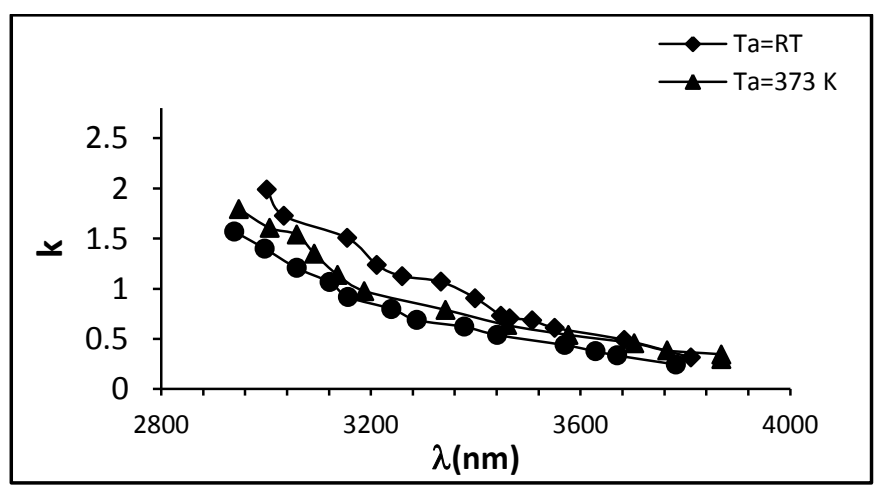

Figure (4): Extinction coefficient as a function of wavelength for Te films at different annealing temperatures

The extinction coefficient (imaginary part of the refractive index), which is related to the exponential decay of the wave as it passes through the medium can be determined by using equation[7]. $\mathrm{k}=\frac{\alpha \lambda}{4 \pi}$

$\lambda:$ is the wavelength of the incident radiation

The relation between the extinction coefficient and wavelength for $\mathrm{Te}$ films at different annealing temperatures is shown in Fig.(4) .From this figure show that the extinction coefficient $(\mathrm{k})$ takes the similar behavior of the corresponding absorption coefficient as shown in Fig.(3).This is attributed to the same reason mentioned previously in the absorption coefficient.

The optical energy gap is given by equation [8] :

$$
\alpha h v=B(h v-\text { Eopt }) 1 / 2 \text {. }
$$

where $\mathrm{B}$ is the constant involving the properties of the bands.

The direct optical energy gaps value (Eop) for Te films have been determined. A plot of ( $\alpha$ hv) 2 versus ho for Te with different annealing temperatures is shown in Fig.(5).The plot is linear indicating the direct band gap of the films. Extrapolation of the linear of the line to the hv axis gives the band gap. The value of the optical energy gap increases with increase of annealing temperature for all samples due to the improved structure of the films. This result is in agreement with[9]

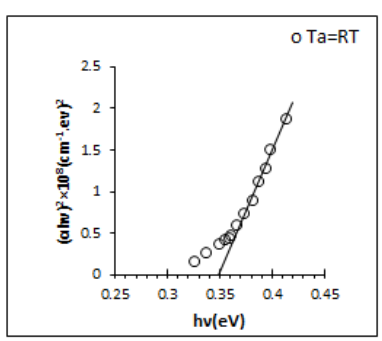

(a)

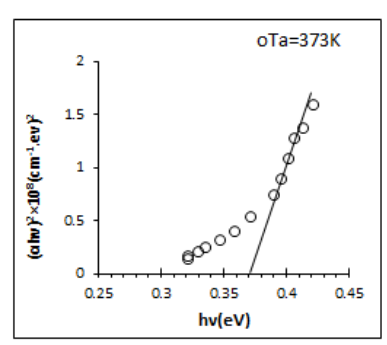

(b)

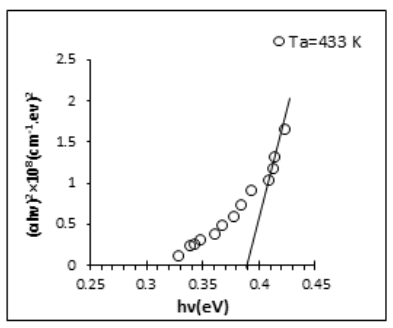

(c)

Figure (5): Plot of $(\alpha h v)^{2}$ as a function of hv for Te films at various annealing $(a=R T, b=373 \mathrm{~K}, c=433 \mathrm{~K})$ 
Table (1): The optical energy gap of Te films at thickness $(0.4 \mu \mathrm{m})$ and different annealing temperature.

\begin{tabular}{|c|c|c|}
\hline $\begin{array}{c}\text { Thickness } \\
(\mu \mathrm{m})\end{array}$ & $\mathrm{T}_{\mathrm{a}} \mathrm{K}$ & $\mathrm{Eg}(\mathrm{eV})$ \\
\hline \multirow{4}{*}{0.4} & $\mathrm{RT}$ & 0.35 \\
\cline { 2 - 3 } & 373 & 0.37 \\
\cline { 2 - 3 } & 433 & 0.39 \\
\hline
\end{tabular}

The refractive index value can be calculated from the formula [10]:

$$
\mathrm{n}=\left(\frac{4 \mathrm{R}}{(\mathrm{R}-1)^{2}}-\mathrm{k}^{2}\right)^{\frac{1}{2}}-\frac{(\mathrm{R}+1)}{(\mathrm{R}-1)}
$$

where $\mathrm{R}$ is the reflectance.

The variation of the refractive index as a function of the wavelength for $\mathrm{Te}$ thin films at different annealing temperatures is shown in Fig.(6), it can be notice the increment of refractive index with wavelength for all specimen at low wavelength then began to decrease as wavelength increases, this may be caused by high absorbance of the films at low wavelength. Also indicate that the decreases with the increasing of annealing temperature due to the increase of the crystallite size with the increase of the annealing temperature.

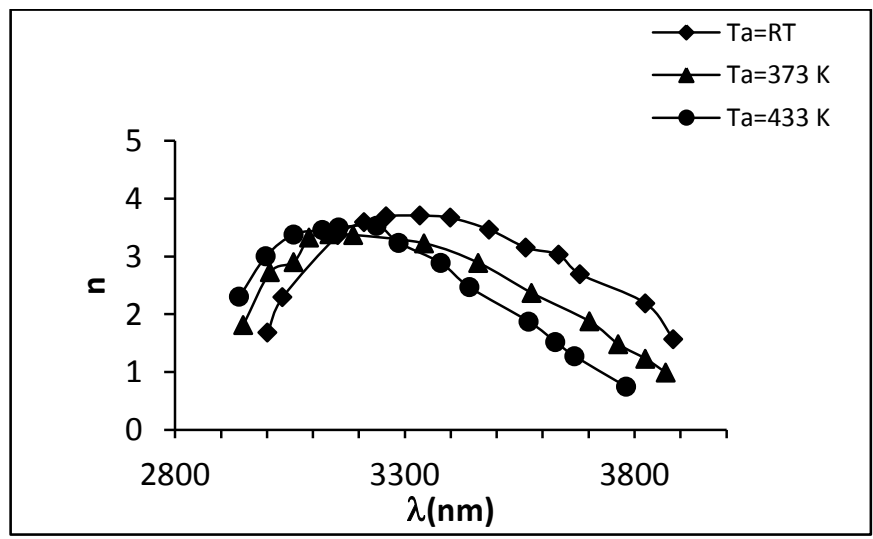

Figure (6): Refractive index as a function of wavelength for Te films at different annealing temperatures and thicknesses $0.4 \mu \mathrm{m}$

\section{Conclusions}

Thin films of Tellurium have been deposited by thermal evaporation technique on glass substrate, From transmittance spectrum in the Infrared region. The transmittance increases with increasing of annealing temperature, Absorption coefficient $(\alpha)$ decreases with increasing of $\mathrm{T}_{\mathrm{a}}$ for all films, absorbance spectra it was observed that the optical transition in the Te thin films is an direct transition and the value of the optical energy gap increases with increasing of $\mathrm{T}_{\mathrm{a}}$ for all samples.

Most of optical characteristics can be improved by annealing of Te film.

\section{References}

[1] Lalla Aicha Ba, Mandy D. oring, Vincent Jamier and Claus Jacob ,Org. Biomol. Chem., 2010, 8, 4203-4216.

[2] K.Wei, D.P.Machewirth, J.Wenzel, G.H.Sigel, J.Non-Cryst Solids, 1995,182, 257.

[3] Mayu.T,Gongzhu.Q, Xuwei.H, Trans. Nonferrous Met. Soc. China. 2006, 16.

[4] F.Habashi, Handbook of Extractive Metallurgy, 1997, v.3.

[5] Burra. R, Determinationof selenium and tellurium oxyanion toxicity, detection of metalloid-containing headspace compounds. A Thesis of Science (Chemistry), 2009, Sam Houston State University.

[6] Tariq J. Alwan and Abbas.K.Hassen,Um-Salama Science Journal, 2008, V.5,1.

[7] Jenkins, F. A. and, H. E. White, Fundamentals of Optics, 1981,McGraw-Hill, New York, pp.234.

[8] L.Kazmarski and A.H.Clark, Polycrystalline and Amorphouse Thin Films and Device, 1980, Edited by Lawrence Academic Press, New York .

[9]P. Ott, J.R Günter, Thin Solid Films, (2000), $366,100$.

[10] S.M.Ahmed, L. A.Latif, A.KH.Salim, Journal of Basrah Researches (Sciences). 2011, 37, N. 3A. 